Research Paper

\title{
CTGF Contributes to the Development of Posterior Capsule Opacification: an in vitro and in vivo study
}

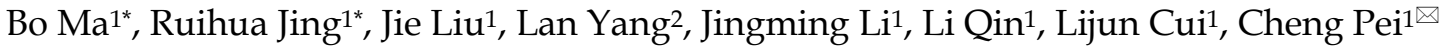 \\ 1. Department of Ophthalmology, First Affiliated Hospital of Xi'an Jiaotong University, Xi'an, Shaanxi, China. \\ 2. Ningbo Medical Center Lihuili Eastern Hospital, Ningbo, Zhejiang, China. \\ *Co-first authors \\ $\triangle$ Corresponding author: Cheng Pei. (email: peich71@163.com). \\ ( ) Ivyspring International Publisher. This is an open access article distributed under the terms of the Creative Commons Attribution (CC BY-NC) license \\ (https://creativecommons.org/licenses/by-nc/4.0/). See http://ivyspring.com/terms for full terms and conditions.
}

Received: 2017.11.20; Accepted: 2018.02.20; Published: 2018.03.11

\begin{abstract}
Connective tissue growth factor (CTGF) is a crucial factor that plays a major role in the process of posterior capsule opacification (PCO). However, the effects of CTGF on the proliferation and migration of lens epithelial cells (LECs) and on the mechanism of the epithelial mesenchymal transition (EMT) and extracellular matrix (ECM) in human lens epithelial cells (HLECs) as well as the effects of shRNA-mediated CTGF knockdown on the development of PCO in rats remain unclear. In the present study, we found that CTGF promoted EMT, proliferation, migration and the expression of p-ERK $1 / 2$ protein in HLECs but exerted little effect on the expression of p-p38 and $\mathrm{P}-J N K 1 / 2$ proteins. MEK inhibitor U0126 effectively restrained the CTGF-induced expression of $\alpha$-smooth muscle actin ( $\alpha-S M A)$, fibronectin (Fn) and type I collagen (COL-1) in HLECs. CTGF knockdown effectively postponed the onset of $\mathrm{PCO}$ in the rats and significantly reduced the expression of $\alpha-S M A$ in the capsule. In conclusion, CTGF contributed to the development of PCO presumably by promoting proliferation, migration of LECs, EMT specific protein expression and ECM synthesis in HLECs, which is dependent on ERK signalling. Furthermore, blocking CTGF effectively inhibited PCO in the rats and the EMT specific protein expression in the lens capsule.
\end{abstract}

Key words: CTGF, Posterior Capsule Opacification, Epithelial Mesenchymal Transition, Extracellular Matrix, ERK signalling

\section{Introduction}

Posterior capsule opacification (PCO) is the most common postoperative complication after extracapsular cataract extraction (ECCE) or phacoemulsification [1]. The main cause of PCO is the transdifferentiation, proliferation, migration and collagen production of residual lens epithelial cells (LECs) in the capsule.

Studies have shown that multiple regulatory factors are involved in the occurrence and development of PCO [2], including connective tissue growth factor (CTGF). CTGF is a crucial factor that was discovered in recent years and plays major roles in mitosis, fibroblastic proliferation, collagen synthesis, and cell adhesion, migration, and fibrosis [3-7]. CTGF promotes the fibrosis of LECs, trabecular meshwork cells and retinal pigment epitheliums. Therefore, CTGF is a major cause of many intraocular pathological changes [8-10], and it is closely associated with transforming growth factor- $\beta$ (TGF- $\beta$ ). Our previous studies found that CTGF is a downstream effector of TGF- $\beta 2$ and participates in EMT and ECM synthesis and pathological changes in various cells, such as vascular smooth muscle cells (VSMCs) and LECs [11-15]. Studies have also found that CTGF induces the synthesis of the EMT-specific marker a-SMA and various ECM proteins, such as COL-1 and Fn by the LECs [16].

Studies have shown that CTGF can activate multiple signalling pathways, including ERK, p38, JNK, ROS, ASK1 and NF-KB, and promote the 
migration and proliferation of human Tenon's capsule fibroblasts, hepatic stellate cells (HSCs), hepatoma carcinoma cells, cardiomyocytes and glioma cells [17-22]. Our former research findings showed that CTGF is under the regulation of the TGF- $\beta / \mathrm{Smad}$ signalling pathway. However, signalling pathways downstream of CTGF that are involved in the migration of HLECs and regulation of the production of EMT and ECM remain uncharacterized in HLECs.

After cataract surgery, the blood-aqueous barrier is damaged, which increases the expression of a cohort of growth factors and cytokines (CKs), including TGF- $\beta$, FGF and PDGF in the aqueous humour, which can stimulate EMT [23]. TGF- $\beta$ has been shown to induce EMT in rat LECs; therefore, it plays an important role in rat anterior subcapsular cataracts (ASCs) and PCO [24]. AKT inhibitors have been found to effectively prevent canine PCO [25]. However, previous studied have not determined whether the specific inhibition of CTGF expression could effectively inhibit PCO.

In this study, we first tested the effects of CTGF on the capsule bag model, the activation of ERK, p38 and FAK signalling pathways and the migration ability of HLECs. Rat PCO models were then established to examine the impact of CTGF depletion on PCO development in vivo.

\section{Materials and Methods}

\section{Culture and treatment of HLECs}

The HLECs line SRA01/04 was gifted from the Ophthalmology Institute of the Fourth Military Medical University. A total of $1 \times 10^{6}$ cells within 20 passages were seeded into a culture flask with DMEM containing $10 \%$ foetal bovine serum (FBS). The culture medium was replaced with serum-free DMEM when the cells approached $70 \%$ confluence, and the cells were cultured for $24 \mathrm{~h}$. The cells in the experimental group were then treated with $3 \mathrm{~mL}$ of serum-free medium containing CTGF at $60 \mu \mathrm{g} / 1$ for different durations $(0,5,15,30,60,120,240,480,960 \mathrm{~min}$ and 24 h) before the cells were harvested for further analysis. Control group cells were treated with an equal volume of medium because all reagents were water soluble.

\section{Making the Capsular Bag Model}

Ten eyes of 5 pigs were obtained from an abattoir. Cornea was cut along the limbus. After the cornea was removed, capsular bags were prepared by hydrodissection followed by continuous curvilinear capsulorhexis. Then, separated lens from iris and suspensory ligament. The original shape of the capsular was maintained by IOL (OcuLens, RAFI Systems Inc., California, USA) into the capsular bag.
After treatment with CTGF at $60 \mu \mathrm{g} / 1$ or no treatment, the capsular bags were incubated in MEM (CM10010, M\&C GENE TECHNOLOGY LTD., Beijing, China) medium for 14 days in an atmosphere of $5 \% \mathrm{CO}_{2}$ at $37^{\circ} \mathrm{C}$. The medium was changed every 48 hours. LECs proliferation was observed with an inverted microscope (Eclipse Ti, Nikon, Japan). The model was established, and the observation replicated three times in each treatment.

\section{Transwell $\circledast$ migration assays}

Transwell ${ }^{\circledR}$ migration experiments were performed with 24 well plates with Matrigel ${ }^{\mathrm{TM}}$ - coated chambers $(8 \mu \mathrm{m}$ pore size) from BD Biosciences. Cells were grown to $70 \%$ confluence and serum starved for $24 \mathrm{~h}$. Following detachment with trypsin, cells were washed with PBS, resuspended in serum - free medium and $1 \times 10^{4}$ cells were added to the upper chamber. Serum-free medium containing $60 \mu \mathrm{g} / 1$ CTGF, $60 \mu \mathrm{g} / 1 \mathrm{CTGF}+20 \mu \mathrm{M}$ U0126 and with the corresponding amount of DMSO as control were added to the bottom wells of the chambers. After 24 and $48 \mathrm{~h}$, the cells that had not migrated were removed from the upper face of the filters using cotton swabs, and the cells that had migrated were fixed and stained with crystal violet solution.

\section{Wound Healing assay}

The Culture-Insert 2 Well provides two cell culture reservoirs which has a special sticky and bio-compatible surface at the bottom side and are separated by a $500 \mu \mathrm{m}$ thick wall. A cell suspension of HLECs at $3 \times 10^{5}$ cells $/ \mathrm{ml}$ for $70 \mu \mathrm{l}$ were seeded in the designated areas and then cultured at $37^{\circ} \mathrm{C}$ in a humidified atmosphere containing $5 \% \quad \mathrm{CO}_{2}$. When cells were grown to $90 \%$ confluence, gently removed the Culture-Insert 2 Well by using sterile tweezers. The cells in the experimental group were then treated with serum-free medium containing $60 \mu \mathrm{g} / \mathrm{l}$ CTGF, 60 $\mu \mathrm{g} / 1 \mathrm{CTGF}+20 \mu \mathrm{M}$ U0126 and with the corresponding amount of DMSO as control for $48 \mathrm{~h}$. The cells migration were observed by an inverted microscope. The images of wound areas are analysed by Image J software.

\section{Quantitative Real-time PCR and Western blot}

The total RNA extraction, reverse transcription, the qPCR reaction and Western-blot were performed as our previous experiments. The PCR primers were designed and synthesized by $\mathrm{TaKaRa}$ Biotechnology (TAKARA BIO INC., Japan) as shown in table 1. The antibody were shown as follow: a-SMA (ab5694, Abcam, UK), Fn (ab2413, Abcam, UK), COL-1 (AB745, Millipore, USA), p-ERK1/2 (\#4370, CST, USA), ERK1/2 (\#4695, CST, USA), p-p38 (\#4511, CST, USA), 
p38 (\#41666, CST, USA), p-JNK1/2(\#4668, CST, USA), JNK1/2 (\#9258, CST, USA), and $\beta$-actin (\#4970, CST, USA), goat anti-rabbit IgG secondary antibody (\#L3012, SAB, USA).

Table 1. Primers used in qPCR. $\alpha$-SMA: $\alpha$-smooth muscle actin; Fn: fibronectin; COL-1: type I collagen.

\begin{tabular}{lll}
\hline Gene & Forward $\left(\mathbf{5}^{\prime}-\mathbf{3}^{\prime}\right)$ & Reverse $\mathbf{( 5}^{\prime} \mathbf{-}^{\prime} \mathbf{)}$ \\
\hline a-SMA & GACAATGGCTCTGGGCTCT & CTGTGCTTCGTCACCACGTA \\
& GTAA & \\
Fn & CAGGATCACTTACGGAGA & GCCAGTGACAGCATACACAGT \\
& AACAG & G \\
COL-1 & TCTAGACATGTTCAGCTTT & TCTGTACGCAGGTGATTGGTG \\
& GTGGAC & \\
\multirow{2}{*}{-actin } & TGGCACCCAGCACAATGA & CTAAGTCATAGTCCGCCTAGA \\
& A & AGCA \\
\hline
\end{tabular}

\section{PCO modelling}

The animal care, handling and surgical procedures were in accordance with the relevant guidelines and regulations. All of the experimental protocols were approved by the Ethics Committee of Xi'an Jiao Tong University. A total of 70 healthy adult SD rats (provided by the Animal Experimentation Centre affiliated with the School of Medicine, Xi'an Jiaotong University) with an average weight of $200 \mathrm{~g}$ and a gender ratio of 1:1 were selected for the experiment. Pre-examinations were conducted on the rats under a slit lamp to ensure that the rats were free from eye diseases, such as corneal conditions or cataracts. The operation was performed on the left eye only. The target sequences for the rat CTGF.shRNA were as follows: GCAGTTGCAAATACCAGTGCA. A nonsilencing shRNA (sequence: siNC, TTCTCCGAACGTGTCACGT) was used as a control in all shRNA transfection experiments. The rats were divided randomly into 5 groups as follows: Group A (one intracameral injection of CTGF.shRNA AAV complex), Group B (two intracameral injections of the CTGF.shRNA AAV complex), Group C (three intracameral injections of the CTGF.shRNA AAV complex), Group D (one intracameral injection of nonsilencing shRNA (NC.shRNA) AAV), Group E (two intracameral injections of NC.shRNA), Group F (three intracameral injections of NC.shRNA) and Group G (balanced salt solution (BSS) control). The experiment was performed in a sequence of steps. First, an intraperitoneal injection of $700 \mu 110 \%$ chloral hydrate was administered as anaesthesia. The disinfecting drape was placed, and oxybuprocaine (Santen Pharmaceutical Co., Ltd.) was applied to the conducting surface as anaesthesia. A $2.0-\mathrm{mm}$ paracentesis knife (Alcon US) was used on the 11-point corneal surface. A viscoelastic agent (Tianjin Jingming New Technological Development Co., Ltd.) was used for the formation of the anterior chamber, and a discission needle was performed capsulectomy. Hydrodissection was performed, and the lens nucleus was delivered. A double-gun tube (66 VISION TECH Co., Ltd.) was employed to suction the residual cortex of the lens, $2 \mu$ l CTGF shRNA (Shanghai Neuron Biotech) was injected into the capsular bag, and $5 \mu 1$ dexamethasone $+5 \mu \mathrm{l}$ tobramycin $+5 \mu \mathrm{l}$ atropine was injected peribulbarly. One needle was used to suture the corneal incision. After surgery, the animals were fed routinely and received eye drops containing levofloxacin (Santen Pharmaceutical Co., Ltd.) four times daily, and TobraDex (Alcon US) was administered twice daily. The formation of PCO was observed under the slit lamp microscope. The rats were sacrificed on the sixtieth day, and their eyeballs were retrieved.

\section{Immunostaining}

Immunostaining was performed as our previous study [26]. The primary antibodies as follow: a-SMA, ab7817(the capsular bag) and ab5694 (The paraffins sections), abcam, UK; CTGF, ab6992, abcam, UK). Secondary antibodies as follow: fluorescein conjugated IgG (Alexa Fluor goat anti-mouse IgG, 1:400, Invitrogen); Strept-Avidin Biotin Complex (sp-9000, ZSGB-BIO, China).

\section{Statistical analysis}

The GraphPad Prism 5.0 statistics software was employed to perform all of the statistical analyses. All of the data are reported as the mean \pm SD from at least three independent in vitro experiments. Statistical comparisons of the Transwell, real-time PCR and Western blot data with the control group were analysed using a one-way ANOVA, whereas differences between groups were compared using Tukey's HSD test. Differences of $p<0.05$ were considered statistically significant.

\section{Results}

\section{CTGF promote LECs proliferation and EMT specific protein in a Capsular Bag Model}

We investigated the impact of CTGF on the EMT and proliferation ability of LECs using a capsular bag model. We cultured capsular bags with or without CTGF. After being incubated with CTGF (Figure 1A), the number of LECs were obvious increased compared with control (Figure 1F). Furthermore, we used immunofluorescence staining to test the expression of a-SMA in the capsular bag. There were few a-SMA proteins in the control. Nevertheless, the expression of a-SMA were significant increased after treatment with CTGF. The results indicated that CTGF could induce LECs EMT specific protein and promote LECs proliferation in capsular bag model. 


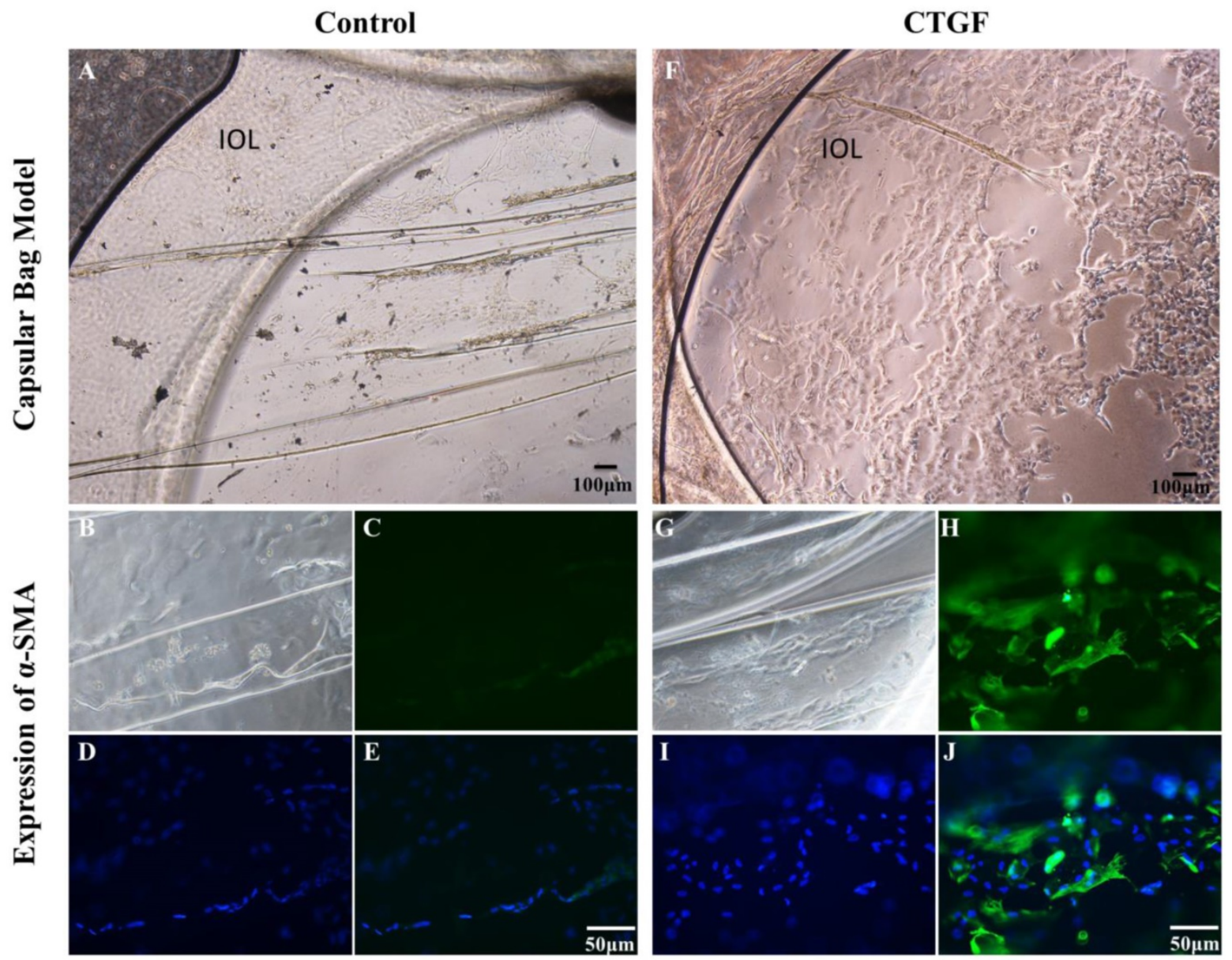

Figure 1. CTGF promotes LECs EMT specific protein and Proliferation in a Capsular Bag Model. A capsular bag model was made and cultured with or without CTGF. A: Control group in 14 day; B, C, D, E: the expression of $\alpha$-SMA in capsular bags in control group; F: Cultured capsular bags with $60 \mu g / /$ CTGF in 14 day; $\mathbf{G}, \mathbf{H}, \mathbf{I}, \mathbf{J}$ : the expression of $\mathrm{Q}-\mathrm{SMA}$ in capsular bags after treatment with CTGF.

\section{Activation of ERK, p38 and JNK signalling pathways by CTGF}

In different tissues, CTGF exerts its biological effects by activating different signalling pathways [27, 28]. However, the signalling pathways that are activated downstream of CTGF during PCO development are not well understood. Therefore, we analysed the effect of CTGF on a variety of signalling pathways. The HLECs were treated with $60 \mu \mathrm{g} / 1$ CTGF for different durations $(0,5,15,30,60,120,240$, 480 and $960 \mathrm{~min})$, and then the expression of the p-ERK1/2, ERK1/2, p-p38, p38, p-JNK1/2 and JNK1/2 proteins were detected by Western blot. The results indicated that the CTGF treatment increased the expression of $\mathrm{p}$-ERK $1 / 2$ in a time-dependent manner, whereas the total ERK1/2 proteins were not changed (Figure $2 \mathrm{~A}, \mathrm{~B})$. However, the expression of p-p38, p-JNK1/2, p38 and JNK1/2 did not show apparent changes (Figure $2 \mathrm{C}, \mathrm{D}$ ). Thus, we concluded that CTGF activates the ERK signalling pathway in HLECs.

\section{Role of ERK signalling pathway in EMT and ECM synthesis in HLECs treated with CTGF}

Our previous studies showed that the CTGF treatment induced the expression of EMT markers and ECM protein synthesis in HLECs [11]. Because CTGF activates the ERK signalling pathway in HLECs, we wondered whether the ERK signalling pathway was involved in the CTGF-induced expression of EMT markers and ECM proteins in HLECs. The HLECs were treated with the MEK inhibitor $20 \mu \mathrm{M}$ U0126 to block the ERK signalling pathway and then treated with CTGF for $24 \mathrm{~h}$. Consistent with prior observations, the mRNA and protein expression of a-SMA, Fn and COL-1 increased significantly after treatment with $60 \mu \mathrm{g} / 1$ CTGF compared with that of the control group. Nonetheless, the induction of a-SMA, Fn and COL-1 by CTGF at both the mRNA and protein levels was significantly inhibited by blocking the ERK signalling pathway with U0126 (Figure 3). 
A CTGF $60 \mu \mathrm{g} / 1 \quad 0 \quad 5 \quad 153060120240480960(\mathrm{~min})$
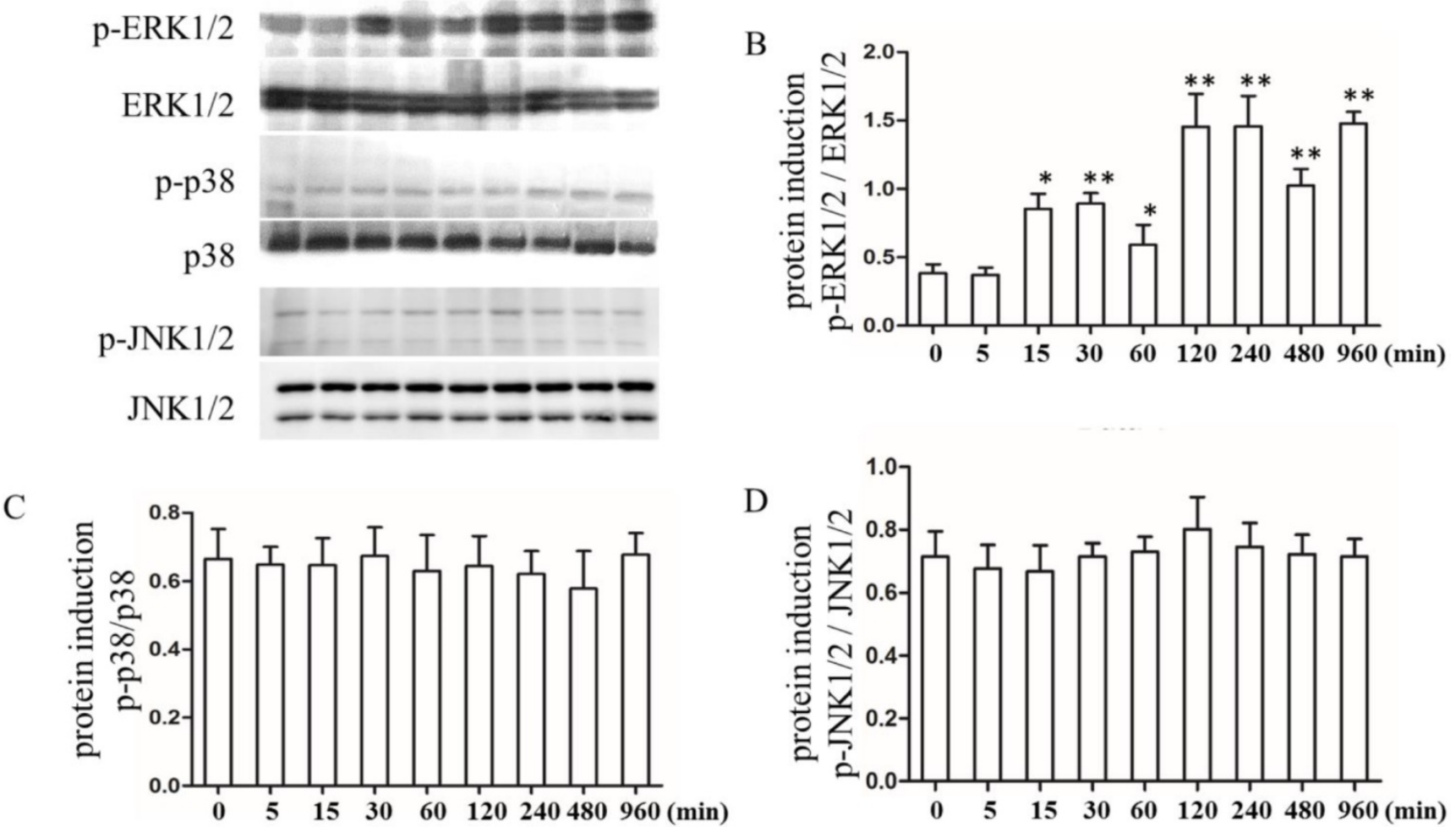

Figure 2. Effects of the CTGF treatment on the ERK, p38 and JNK signalling pathways. HLECs were treated with $60 \mu \mathrm{g} / \mathrm{l}$ CTGF for different durations $(0,5,15,30,60,120,240,480$ and $960 \mathrm{~min})$. A: Expression of p-ERK1/2, ERK1/2, p-p38, p38, p-JNK1/2 and JNK1/2 proteins detected by Western blot; B: Quantitation of the p-ERK levels as shown in A (*p<0.05 or ** $p<0.01)$; $\mathbf{C}$ and $\mathbf{D}$ : Quantitation of the p-p38 and p-JNK levels as shown in A.

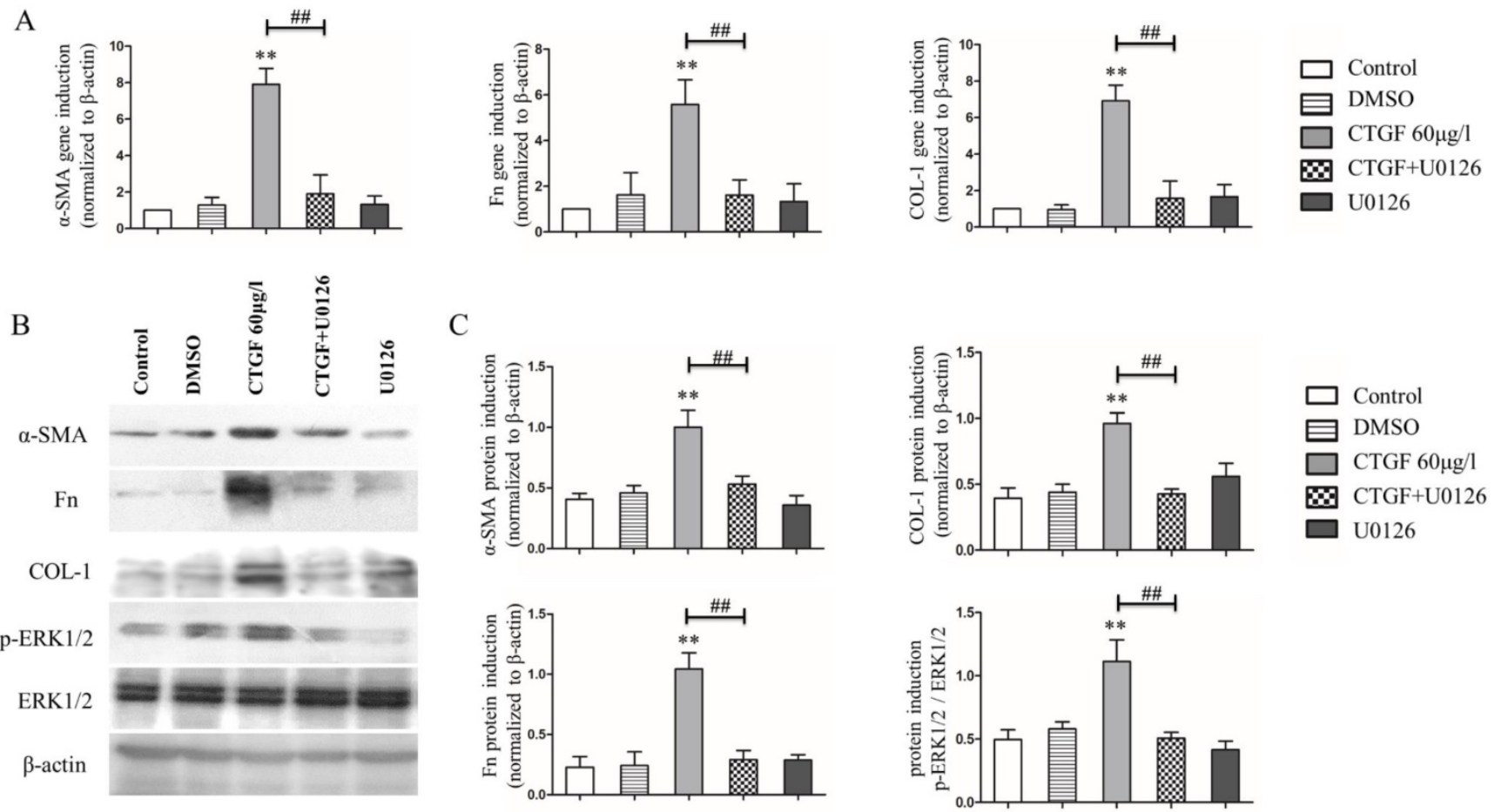

Figure 3. Effects of the MEK inhibitor U0126 on CTGF-induced HLEC expression of a-SMA, Fn and COL-1. A: mRNA levels of $\alpha$-SMA, Fn and COL-1 in the HLECs treated with or without CTGF or U0126, ** $p<0.01$ versus the control; the difference between the group treated with CTGF and CTGF+U0126 was significant $p<0.01$. B: Protein expression of $\alpha$-SMA, Fn and COL-1 in the HLECs treated with or without CTGF or U0126. C: Quantitation of the Western blot results in B** $p<0.01$ versus the control; the difference between the group treated with CTGF and CTGF+U0126 was significant \# $p<0.01$. 


\section{Effect of the CTGF and ERK signalling pathways on HLECs migration}

HLECs migration is one of the most important contributors to PCO development. Studies have shown that CTGF can promote migration in a variety of cells $[29,30]$. However, the effect of CTGF on the migration of HLECs is still unknown. We investigated the impact of CTGF on the migration ability of HLECs using Transwell ${ }^{\circledR}$ migration assay and Wound-Healing assay. The results showed the number of migrated cells and migration rate of CTGF group were significantly higher than the control group $\left({ }^{* *} p<0.01\right.$, Figure 4$)$. Co-treatment with CTGF and U0126 significantly reduced the number of migrated cells and migration rate compared with the CTGF treatment alone $(\# p<0.01$, Figure 4). Interestingly, the number of migrated cells and migration rate were still significantly higher in the CTGF+U0126 group versus the control group $\left({ }^{*} p<0.05,{ }^{* *} p<0.01\right)$, suggesting other downstream effectors, in addition to ERK signalling may also be involved in the migration-promoting effect of CTGF.

A
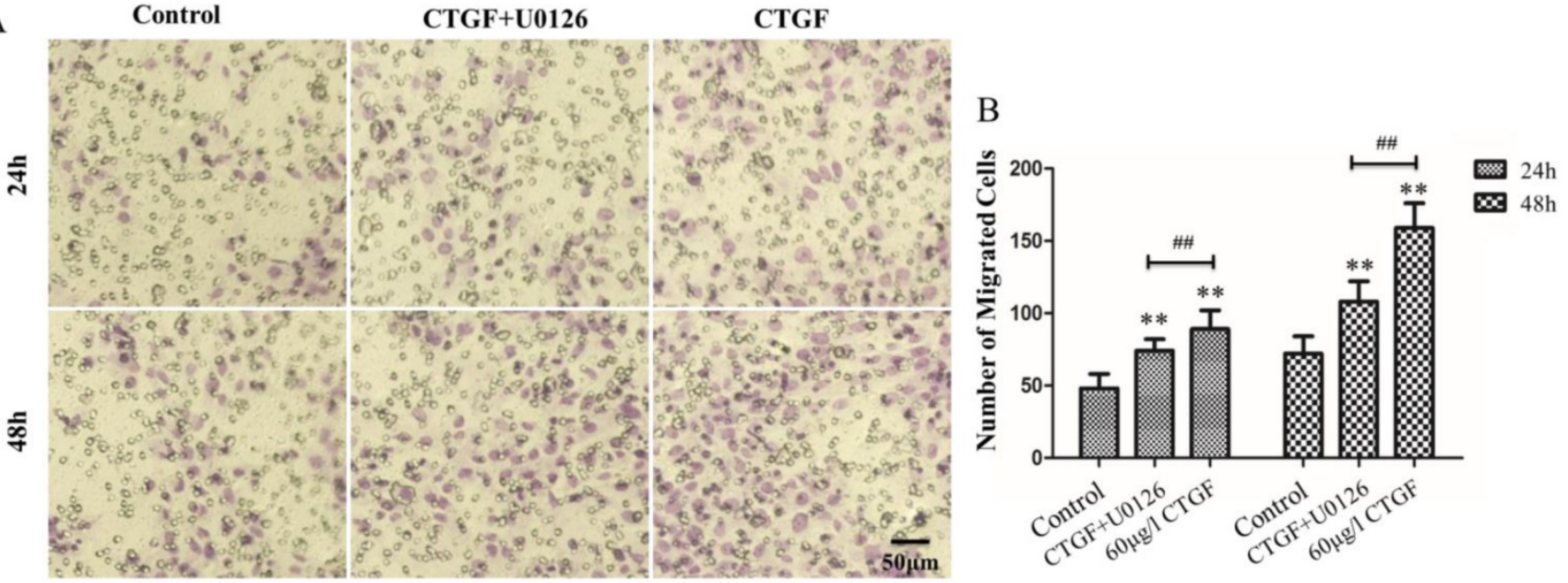

C

$\mathrm{Oh}$

$24 \mathrm{~h}$

$48 \mathrm{~h}$
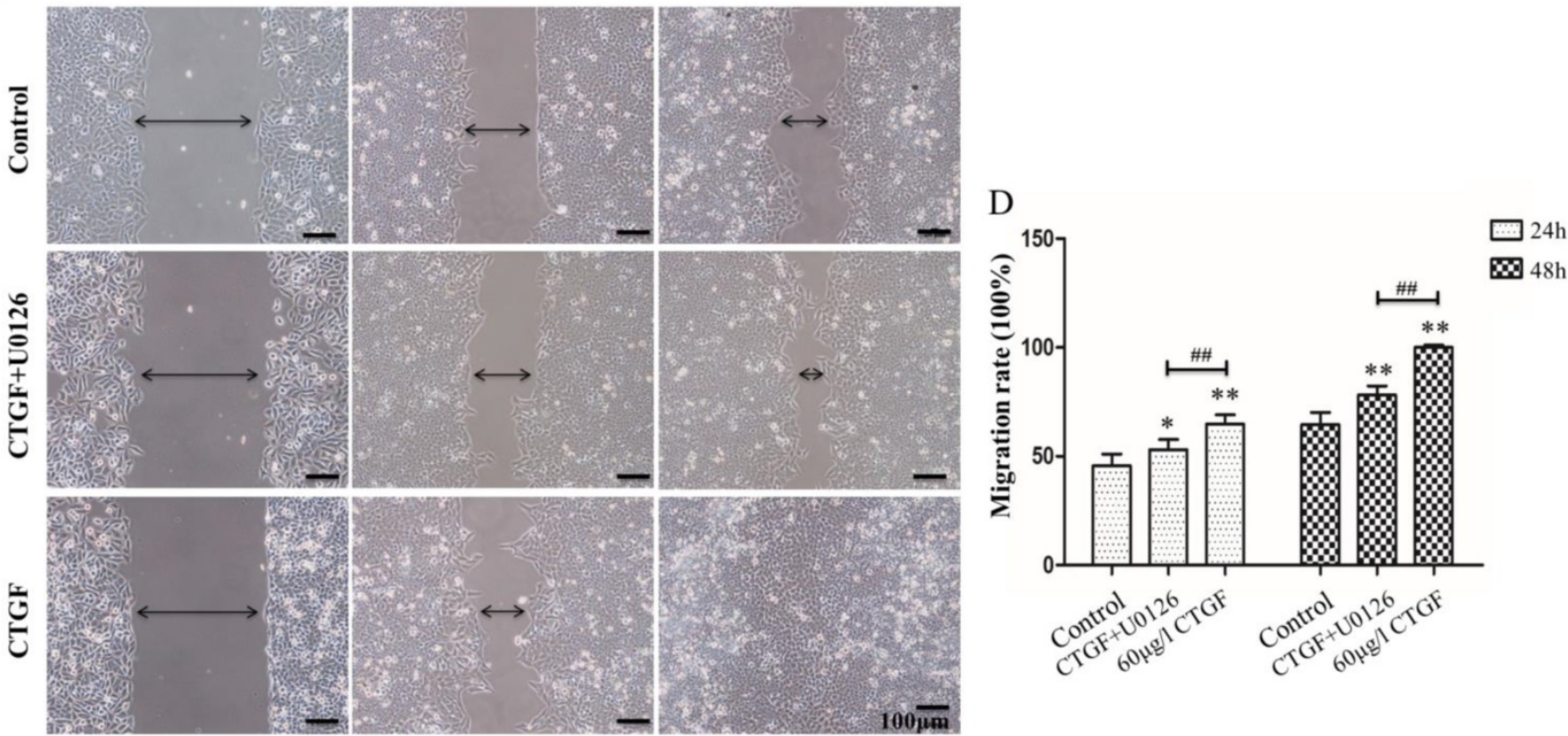

Figure 4. CTGF promoted the migration of HLECs in an ERK signalling-dependent manner. A: HLECs were induced with $60 \mu \mathrm{Hg} / \mathrm{CTGF}$ in the presence or absence of $20 \mu \mathrm{M} \cup 0126$ for $24 \mathrm{~h}$, and the effect on migration was evaluated using the Transwell assay. Scale bar: $50 \mu \mathrm{m}$. B: Quantitation of the Transwell assay, $* *$ $p<0.01$; The migration rates were significant between CTGF and CTGF+U0126 groups at $24 \mathrm{~h}$ and $48 \mathrm{~h} \# p<0.01$. C: HLECs were induced with $60 \mu \mathrm{mg} / \mathrm{CTGF}$ in the presence or absence of $20 \mu \mathrm{M} \cup 0126$ for $24 \mathrm{~h}$ and $48 \mathrm{~h}$, and the effect on migration was evaluated using the wound healing assay. Scale bar: $100 \mu \mathrm{m}$. D: The migration rates were significant in CTGF and CTGF+U0126 groups compared with control at $24 \mathrm{~h}$ and $48 \mathrm{~h} * p<0.05$; The migration rates were significant between CTGF and CTGF+U0126 groups at $24 \mathrm{~h}$ and $48 \mathrm{~h} \# \mathrm{p}<0.01$ 


\section{Effects of specific CTGF silencing on rat PCO}

TGF- $\beta 2$ is considered an important factor in the development of PCO [16], and it plays an important role in maintaining the physiological function of the body; moreover, blocking this factor will cause a series of pathological changes. Thus, a factor that is specific and essential for tissue fibrosis and does not promote severe pathological changes after its expression is blocked must be targeted. CTGF has been shown to act as a downstream effector of TGF- $\beta 2$, which is mainly involved in the process of fibrosis in various tissues [12, 13]. Therefore, we hypothesized that targeting CTGF may delay the onset of PCO or alleviate its symptoms. To test this hypothesis, we injected CTGF.shRNA with an AAV vector to silence the expression of CTGF and observe the associated effects on PCO occurrence in a rat PCO model. The results showed that the BSS group, one intracameral injection, two intracameral injections and three intracameral injections of the NS groups developed PCO after 20 days and opacification became obvious on the 40th day. The capsules of the groups with one or two intracameral injections of CTGF shRNA were transparent on day 40, although partial opacification was observed on day 60 . Remarkably, the capsules of the group with 3 intracameral injections of CTGF shRNA did not show obvious opacification on the 60th day (Figure 5).

Table 2. PCO grading standard.

\begin{tabular}{ll|l}
\hline Grade & Description & Color \\
\hline 0 (none) & $\begin{array}{l}\text { Transparent of capsule } \\
\text { 1 (minimal) }\end{array}$ & $\begin{array}{l}\text { Mild wrinkling, mild homogenous layers, or sheets } \\
\text { of LECs }\end{array}$ \\
2 (mild) & $\begin{array}{l}\text { Honeycomb patterns of PCO, thicker homogenous } \\
\text { layers, and denser fibrosis }\end{array}$ & \\
3 (moderate) & $\begin{array}{l}\text { Classical Elschnigg pearls and of very thick } \\
\text { homogenous layers }\end{array}$ & \\
4 (severe) & $\begin{array}{l}\text { very thick Elschnigg pearls with "darkening effect" } \\
\text { or of any type of severe opacification }\end{array}$ & \\
\hline
\end{tabular}

\section{Assessment of PCO}

The PCO assessment was compared among the seven groups with EPCO software (http://www.epco2000.de/). The PCO grades were shown in table 2. The results showed that the BSS group, one intracameral injection, two intracameral injections and three intracameral injections of the NC.shRNA groups PCO scores were significant higher than three intracameral injections of the CTGF.shRNA AAV group (** $p<0.01$, Figure 6 ). The one intracameral injections of the CTGF.shRNA AAV group PCO score was significant higher than three intracameral injections of the CTGF.shRNA AAV group $\left({ }^{*} p<0.05\right.$, Figure 6$)$. However, there was no significant difference between three intracameral injections of the CTGF.shRNA AAV group and two intracameral injections of the CTGF.shRNA AAV group (Figure 6). Together, our results indicated that specific blockade of CTGF can effectively prevent or delay the occurrence of PCO.

\section{Effects of specific CTGF silencing on the post-cataract expression of CTGF and $\alpha$-SMA protein in the rat lens capsule}

CTGF is rarely expressed in normal tissues, although damage to the blood-aqueous barrier by external forces, such as surgery or trauma, increases the expression of CTGF $[17,31]$. We conducted ECCE on SD rats, sacrificed the rats after 60 days and observed the expression of CTGF and a-SMA protein in the lens capsule using immunohistochemical staining. The results showed that the expression of CTGF and a-SMA protein increased significantly in the BSS control group and the two intracameral injections of the NC.shRNA group (Figure 7B, C, F, $G)$, whereas the expression decreased in the group that had 3 injections of CTGF.shRNA AAV (Figure 7A, E). We did not observe any expression of CTGF and a-SMA protein in the non-surgery group (Figure 7D, H). These results indicated that the CTGF-specific shRNA successfully blocked the surgery-induced upregulation of CTGF and a-SMA in residual rat LECs.

\section{Discussion}

PCO is the most common complication after ECCE or phacoemulsification. The main cause of $\mathrm{PCO}$ is the transdifferentiation, proliferation, migration and collagen production of LECs in the capsule. Currently, YAG is the main treatment for PCO, although intraocular lens injury may occur during the operation, and complications such as macular oedema, elevated intraocular pressure and retinal detachment may occur after surgery. Therefore, developing new approaches to prevent the occurrence of PCO has become a popular research endeavour in recent years, and the role of growth factors and cytokines in fibrosis development has drawn increasing attention. CTGF is an important recently discovered secretory growth factor, and it occurs in a variety of human tissues and organs and exhibits especially high contents in the kidney. In different tissues, CTGF exerts its biological effects via binding with different receptors [32,33]. In-depth studies on the relationship between CTGF and PCO and the functional mechanisms have provided rationales for focusing on CTGF as a potential target for PCO prevention and treatment. 

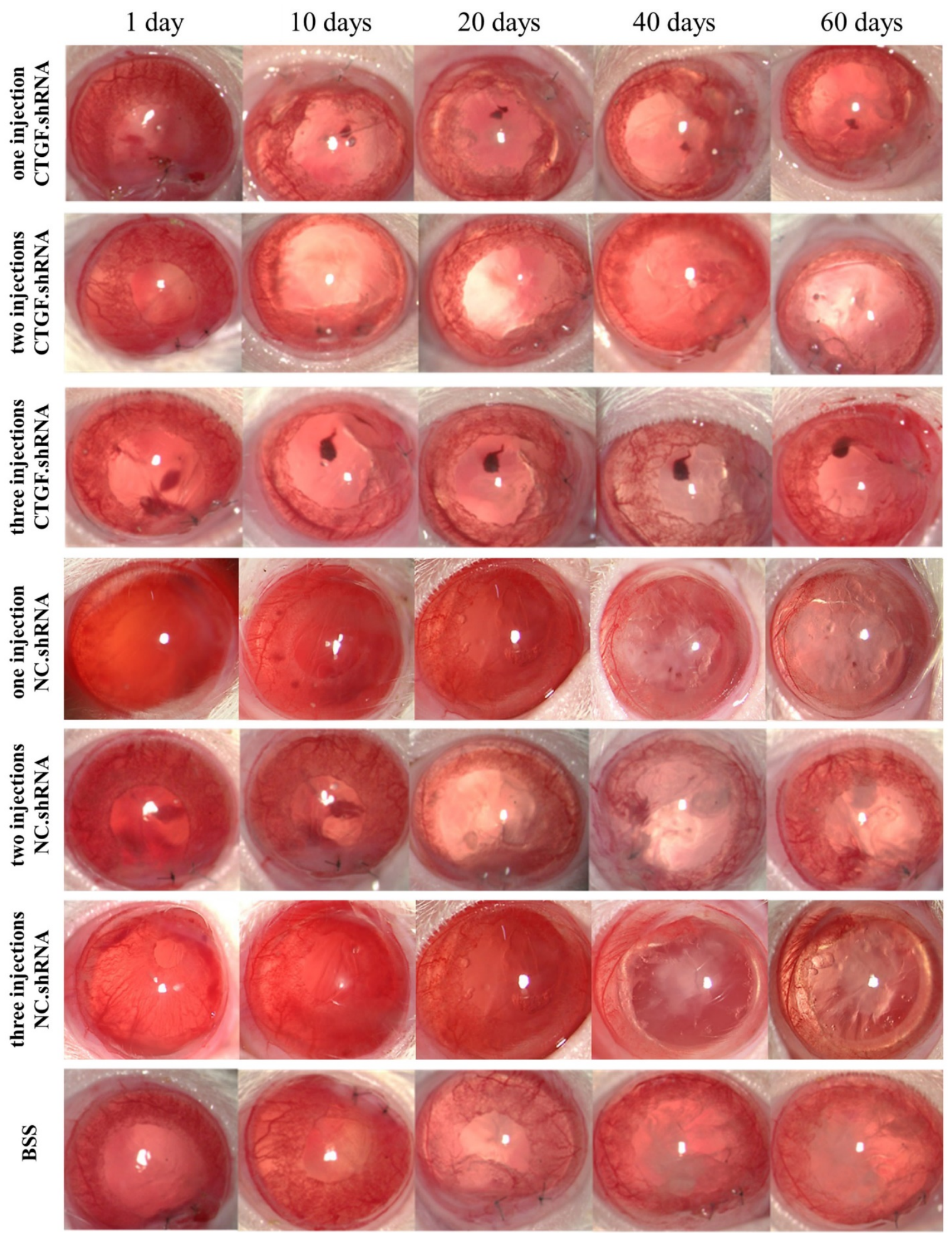

Figure 5. Occurrence of $P C O$ observed in a slit lamp at different times after ECCE. PCO observed at 1-60 days with the 1-3 injections of CTGF.shRNA AAV complex, 1-3 injections of NC.shRNA AAV complex and BSS control group. 


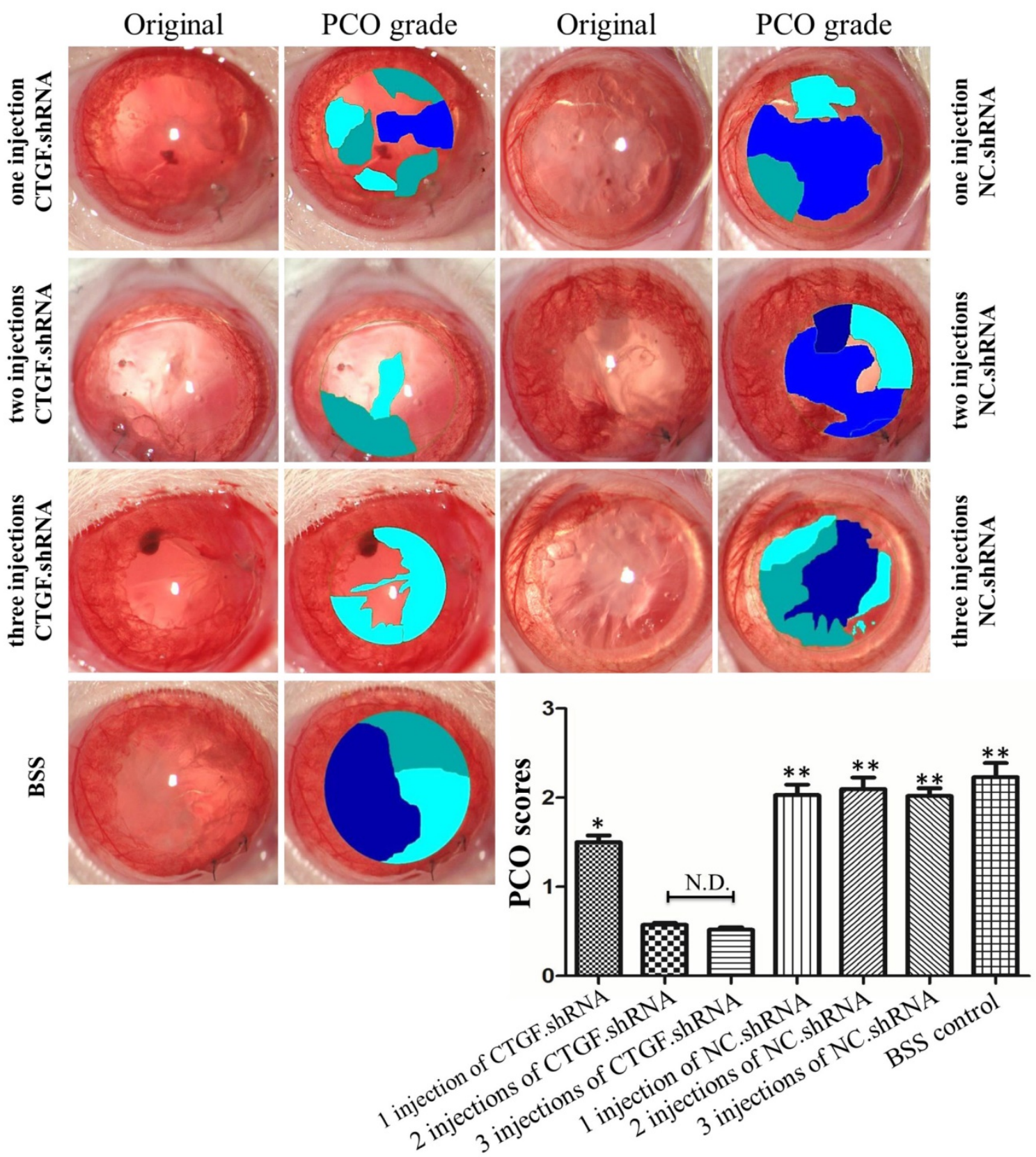

Figure 6. Comparison of PCO scores by EPCO software. PCO grades and scores were statistic by EPCO software in each group. The Significant differences were observed in PCO scores compared with the three intracameral injections of the CTGF.shRNA group, $* p<0.05, * * p<0.01$.

CTGF plays an important role in many eye diseases, such as glaucoma and vitreous retinal disease, and is vital for corneal repair [12, 34-37]. Moreover, CTGF may act as a downstream effector of TGF- $\beta$ to promote fibrosis and stimulate fibroblast proliferation and ECM synthesis, and it also promotes wound healing and tissue fibrosis $[9,38,39]$. The EMT of LECs is the main pathological change associated with PCO [14], which is characterized by cell morphology changes, epithelial phenotype loss, actin reorganization and interstitial cell phenotypes. a-SMA is an important marker of EMT and a key factor in tissue fibrosis. The rearrangement of the actin cytoskeleton and the induction of a-SMA during EMT provide a structural basis for the migration, invasion and contractility of the transdifferentiated cells. As the main ECM components, COL- 1 and Fn are distributed on the cell surface or between cells, where they form a network structure to provide support for the migration of cells. 


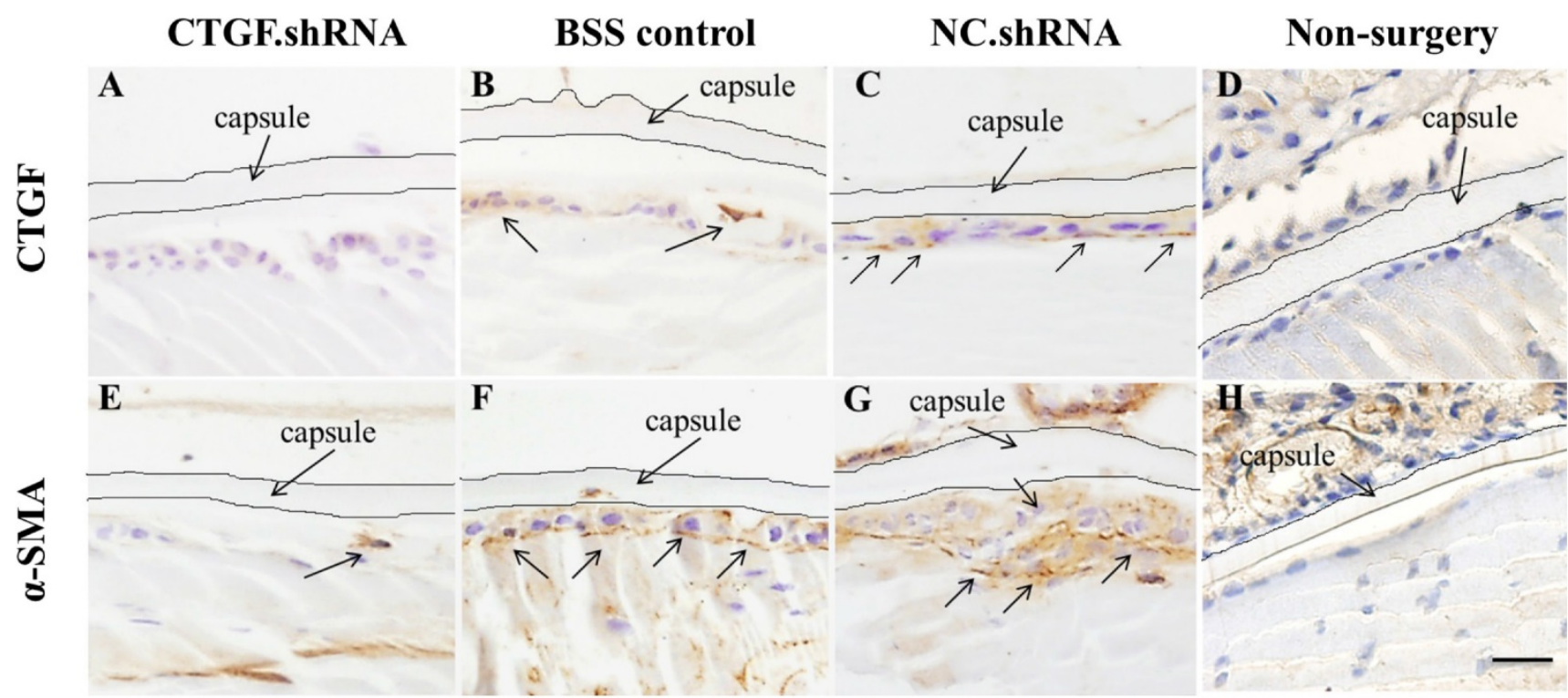

Figure 7. Expression of CTGF and a-SMA proteins in the lens capsule detected by IHC after ECCE. (A, E): Expression of CTGF and $\alpha$-SMA proteins in the group with 3 injections of the CTGF.shRNA AAV complex; (B, F): Expression of CTGF and Q-SMA proteins in the lens capsules of the BSS control group; (C, G): Expression of CTGF and a-SMA proteins in the NC.shRNA group; (D, H): Expression of CTGF and a-SMA proteins in the non-surgery group. Scale bar: $50 \mu \mathrm{m}$.

CTGF played different proliferation effects in different cells, but CTGF could definitely promote fibroblast proliferation [40-42]. In order to better investigate the effects of CTGF on LECs EMT and proliferation, we use a capsular bag model. Our results indicated that LECs happened EMT and proliferation after being induced by CTGF. Furthermore, because some LECs gathered into a mass and happened differentiation, it was difficult to statistics the number of LECs. However, it was also obvious that the number of cells and the expression of a-SMA were significant increased after treatment with CTGF.

The ERK, p38 and JNK signalling pathways are involved in EMT and ECM production of a variety of cells [43-47]. It has been found that CTGF could activate Ras/MEK/ERK/STAT3 pathway. Selective inhibitors of Ras, MEK 1/2, and ERK1/2 individually blocked both CTGF induced cell proliferation, and collagen gel contraction [48]. However, previous studies have not been performed to investigate the relationship of the ERK, p38 and JNK signalling pathways with CTGF-induced EMT specific protein and ECM synthesis in HLECs. Our previous results showed that CTGF can promote EMT and ECM production in HLECs in a Smad signalling-dependent manner [11]. The results of the current study indicated that the ERK pathway but not the p38 or JNK signalling pathways was activated by CTGF and contributed to CTGF-induced EMT specific protein and ECM synthesis in HLECs. This observation is consistent with a previous study in glioma cells [20]. Interestingly, CTGF was found to promote fibrosis in various tissues by activating the TGF- $\beta / \mathrm{Smad}$ signalling pathway, thus implying the presence of a positive feedback loop.

Studies have shown that CTGF promotes the migration and proliferation of HSCs and hepatoma carcinoma cells [5]. In glaucoma, CTGF promotes the migration of human Tenon's capsule fibroblasts, which could be blocked by paclitaxel [21]. However, the effect of CTGF on the migration of HLECs has not been studied. Our present study provides the first evidence that CTGF could enhance the migratory capacity of HLECs. Blocking the ERK signalling pathway effectively decreased the migration of HLECs induced by CTGF, although this migration was not completely inhibited. The pro-migratory effect of CTGF may represent an integrated outcome of the actions of multiple factors and signalling pathways; therefore, the inhibition of one of these factors or pathways cannot block the process completely. Further studies are needed to fully investigate this issue.

Studies have shown that blocking Akt signalling effectively prevented the occurrence of canine PCO, and medicines such as fluorouracil (5-FU), nonsteroidal anti-inflammatory drugs (NSAIDs), diclofenac, doxorubicin and celecoxib could also reduce the occurrence of PCO [49-51]. Our previous study showed that blocking the expression of CTGF effectively inhibited TGF- $\beta 2$-induced EMT and ECM production in HLECs. However, whether specific inhibition of CTGF expression can effectively inhibit PCO is still unclear. Studies have found that inhibiting the expression of CTGF provided a 
meaningful reduction in renal fibrosis [52]. Research has shown that vaccination against CTGF inhibited fibrogenesis, alleviated hepatocyte apoptosis and facilitated hepatic regeneration, and a vaccine against CTGF could be developed into an effective therapeutic measure for hepatic fibrosis [53]. Based on these observations, we conducted further in vivo studies to test whether CTGF silencing by specific shRNA AAV could effectively prevent PCO. Our results showed that the specific silencing of CTGF effectively prevented and delayed the occurrence of PCO and inhibited the induction of the EMT marker a-SMA in rat LECs after surgery. Based on these results, we suggested that CTGF may represent a target for effectively preventing the occurrence of PCO.

This study found that CTGF promoted the EMT specific protein, proliferation and migration of LECs and activated the ERK signalling pathway but not the p38 and JNK signalling pathways. Additionally, our results showed that the ERK signalling pathway played an important role in CTGF-induced EMT and ECM proteins expression in HLECs. The depletion of CTGF effectively postponed the onset of PCO in a rat eye PCO model. This study provides a theoretical basis for further exploring the role of CTGF in the development of PCO, and our findings suggest that CTGF may be a new potential target for the prevention and treatment of PCO.

\section{Acknowledgements}

This study was supported by the fund from the National Natural Science Foundation of China (No. 81470614). We thank the Institute of Neurobiology of the Xi'an Jiaotong University College of Medicine for assisting in the study.

\section{Author Contributions}

C. Pei and L. Qin were responsible for designing the study and performing a critical review of the manuscript. B. Ma and J. M. Li were responsible for designing and performing the study, performing the literature research and writing the manuscript. R. H. Jing, J. Liu and L. Yang were responsible for data acquisition and analysis. L. J. Cui was responsible for the critical review of the manuscript. All authors approved the final version of the manuscript.

\section{Abbreviations}

CTGF: Connective tissue growth factor; a-SMA: a-smooth muscle actin; Fn: fibronectin; COL-1: type I collagen; PCO: posterior capsule opacification; EMT: epithelial mesenchymal transition; ECCE: extracapsular cataract extraction; TGF- $\beta$ : transforming growth factor- $\beta$; LEC: lens epithelial cells; HSCs: hepatic stellate cells; CKs: cytokines; ASCs: anterior subcapsular cataracts; FBS: foetal bovine serum; BSS: balanced salt solution.

\section{Competing Interests}

The authors have declared that no competing interest exists.

\section{References}

1. Pandey S, Apple D, Werner L, Maloof A, Milverton E. Posterior Capsule Opacification : A Review of the Aetiopathogenesis, Experimental and Clinical Studies and Factors for Prevention. Indian J Ophthalmol. 2004; 52: 99-112.

2. Wormstone IM, Wang L, Liu CSC. Posterior capsule opacification. Exp Eye Res. 2009; 88: 257-69.

3. Bagheri A, Soheili Z-S, Ahmadieh H, Samiei S, Sheibani N, Astaneh SD, et al. Simultaneous application of bevacizumab and anti-CTGF antibody effectively suppresses proangiogenic and profibrotic factors in human RPE cells. Mol Vis. 2015; 21: 378-90.

4. Chaqour B, Goppelt-Struebe M. Mechanical regulation of the Cyr61/CCN1 and CTGF/CCN2 proteins. FEBS J. 2006; 273: 3639-49.

5. Hendesi H, Barbe M, Safadi F, Monroy M, Popoff S. Integrin mediated adhesion of osteoblasts to connective tissue growth factor (CTGF/CCN2) induces cytoskeleton reorganization and cell differentiation. Plos One. 2015; 10: e0115325.

6. Lee M, Ghim J, Kim S, Yun Y, Yoo S, Suh P, et al. Functional interaction between CTGF and FPRL1 regulates VEGF-A-induced angiogenesis. Cell Sigal 2015; 27: 1439-48.

7. Yang Z, Sun Z, Liu H, Ren Y, Shao D, Zhang W, et al. Connective tissue growth factor stimulates the proliferation, migration and differentiation of lung fibroblasts during paraquat-induced pulmonary fibrosis. Mol Med Rep. 2015; 12: 1091-7.

8. Khankan R, Oliver N, He S, Ryan SJ, Hinton DR. Regulation of fibronectin-EDA through CTGF domain-specific interactions with TGF $\beta 2$ and its receptor TGF $\beta$ RII. Invest Ophth Vis Sci. 2011; 52: 5068.

9. Iyer P, Maddala R, Pattabiraman PP, Rao PV. Connective tissue growth factor-mediated upregulation of neuromedin $U$ expression in trabecular meshwork cells and its role in homeostasis of aqueous humor outflow. Invest Ophth Vis Sci. 2012; 53: 4952.

10. Junglas B, Kuespert $\mathrm{S}$, Seleem AA, Struller T, Ullmann $\mathrm{S}$, Bösl M, et al. Connective tissue growth factor causes glaucoma by modifying the actin cytoskeleton of the trabecular meshwork. Am J pathol. 2012; 180: 2386.

11. Ma B, Kang Q, Qin L, Cui L, Pei C. TGF- $\beta 2$ induces transdifferentiation and fibrosis in human lens epithelial cells via regulating gremlin and CTGF. Biochem Biophys Res Commun. 2014; 447: 689.

12. Esson DW, Neelakantan A, Iyer SA, Blalock TD, Balasubramanian L, Grotendorst GR, et al. Expression of connective tissue growth factor after glaucoma filtration surgery in a rabbit model. Invest Ophth Vis Sci. 2004; 45: 485.

13. Pei C, Ma B, Kang OY, Oin L, Cui LJ. Effects of transforming growth factor $\beta 2$ and connective tissue growth factor on induction of epithelial mesenchymal transition and extracellular matrix synthesis in human lens epithelial cells. Int J Ophthalmol. 2013; 6: 752-7.

14. Lovicu FJ, Schulz MW, Hales AM, Vincent LN, Overbeek PA, Chamberlain $\mathrm{CG}$, et al. TGFbeta induces morphological and molecular changes similar to human anterior subcapsular cataract. Brit J Ophthalmol. 2002; 86: 220.

15. Wunderlich $K$, Pech $M$, Eberle AN, Mihatsch $M$, Flammer $J$, Meyer $P$. Expression of connective tissue growth factor (CTGF) mRNA in plaques of human anterior subcapsular cataracts and membranes of posterior capsule opacification. Curr Eye Res. 2000; 21: 627.

16. Lee $\mathrm{EH}$, Joo $\mathrm{CK}$. Role of transforming growth factor-beta in transdifferentiation and fibrosis of lens epithelial cells. Invest Ophth Vis Sci. 1999; 40: 2025-32.

17. Gjymishka A, Pi L, Oh SH, Jorgensen M, Liu C, Protopapadakis $\mathrm{Y}$, et al miR-133b Regulation of Connective Tissue Growth Factor: A Novel Mechanism in Liver Pathology. Am J Pathol 2016; 186: 1092.

18. Liu SC, Lee HP, Hung CY, Tsai CH, Li TM, Tang CH. Berberine attenuates $\mathrm{CCN} 2$-induced IL-1 $\beta$ expression and prevents cartilage degradation in a rat model of osteoarthritis. Toxicol Appl Pharm. 2015; 289: 20-9.

19. Nagai N, Klimava A, Lee WH, Izuminagai K, Handa JT. CTGF is increased in basal deposits and regulates matrix production through the ERK (p42/p44mapk) MAPK and the p38 MAPK signaling pathways. Invest Ophth Vis Sci. 2009; 50: 1903.

20. Mendes FA, Coelho Aguiar JM, Kahn SA, Reis AH, Dubois LG, Romão LF, et al. Connective-Tissue Growth Factor (CTGF/CCN2) Induces Astrogenesis and Fibronectin Expression of Embryonic Neural Cells In vitro. Plos One. 2015; 10: e0133689.

21. Chen N, Guo D, Guo Y, Sun Y, Bi H, Ma X. Paclitaxel inhibits cell proliferation and collagen lattice contraction via TGF- $\beta$ signaling pathway in human tenon's fibroblasts in vitro. Eur J Pharmacol. 2016; 777: 33. 
22. Jeong MH, Kim SJ, Kang H, Park KW, Park WJ, Yang SY, et al. Cucurbitacin I Attenuates Cardiomyocyte Hypertrophy via Inhibition of Connective Tissue Growth Factor (CCN2) and TGF- $\beta$ /Smads Signalings. Plos One. 2015; 10: e0136236.

23. Iyengar L, Patkunanathan B, Mcavoy JW, Lovicu FJ. Growth factors involved in aqueous humour-induced lens cell proliferation. Growth Factors. 2009; 27: 50 .

24. Iongh RUD, Wederell E, Lovicu FJ, Mcavoy JW. Transforming Growth Factor- $\beta$-Induced Epithelial-Mesenchymal Transition in the Lens: A Model for Cataract Formation. Cells Tissues Organs. 2005; 179: 43.

25. Chandler HL, Webb TR, Barden CA, Thangavelu M, Kulp SK, Chen CS, et al. The effect of phosphorylated Akt inhibition on posterior capsule opacification in an ex vivo canine model. Mol Vis. 2010; 16: 2202-14.

26. Zhang Z, Hu F, Liu Y, Ma B, Chen X, Zhu K, et al. Activation of type 5 metabotropic glutamate receptor promotes the proliferation of rat retinal progenitor cell via activation of the PI-3-K and MAPK signaling pathways. Neuroscience. 2016; 322: 138-51.

27. Shafieian M, Chen S, Wu S. Integrin-linked kinase mediates CTGF-induced epithelial to mesenchymal transition in alveolar type II epithelial cells. Pediatr Res. 2015; 77: 520.

28. Zhu X, Jing Z, Zhen Z, Sheng J, Jiang W, Liu J, et al. Epithelial derived CTGF promotes breast tumor progression via inducing EMT and collagen I fibers deposition. Oncotarget. 2015; 6: 25320-38.

29. Guo Y, Lu X, Wang H. Downregulation of miR-18a induces CTGF and promotes proliferation and migration of sodium hyaluronate treated human corneal epithelial cells. Gene. 2016; 591: 129-36.

30. Kim MJ, Gloss BS, Rajmohan M, Chang DK, Colvin EK, Jones MD, et al. Connective tissue growth factor as a novel therapeutic target in high grade serous ovarian cancer. Oncotarget. 2015; 6: 44551-62.

31. Yuan HP, Li XH, Yang BB, Shao ZB, Yan LP. [Expression of connective tissue growth factor after trabeculectomy in rabbits]. [Zhonghua yan ke za zhi]. 2009; 45: 168 .

32. Song JJ, Aswad R, Kanaan RA, Rico MC, Owen TA, Barbe MF, et al. Connective tissue growth factor (CTGF) acts as a downstream mediator of TGF- $\beta 1$ to induce mesenchymal cell condensation. J Cell Physiol. 2010; 210: 398-410.

33. Gao R, Brigstock DR. A novel integrin alpha5beta1 binding domain in module 4 of connective tissue growth factor (CCN2/CTGF) promotes adhesion and migration of activated pancreatic stellate cells. Gut. 2006; 55: 856.

34. Robinson PM, Smith TS, Patel D, Dave M, Lewin AS, Pi L, et al. Proteolytic processing of connective tissue growth factor in normal ocular tissues and during corneal wound healing. Invest Ophthalmol Vis Sci. 2012; 53: 8093-103.

35. Setten GBV, Blalock TD, Grotendorst G, Schultz GS. Detection of connective tissue growth factor (CTGF) in human tear fluid: preliminary results. Acta Ophthalmol. 2010; 81: 51-3.

36. Meyer P, Wunderlich K, Kain HL, Prünte C, Flammer J. Human Connective Tissue Growth Factor mRNA Expression of Epiretinal and Subretinal Fibrovascular Membranes: A Report of Three Cases. Ophthalmologica. 2002; 216: 284-91.

37. Kita T, Hata Y, Kano K, Miura M, Nakao S, Noda Y, et al. Transforming growth factor-beta2 and connective tissue growth factor in proliferative vitreoretinal diseases: possible involvement of hyalocytes and therapeutic potential of Rho kinase inhibitor. Diabetes. 2007; 56: 231.

38. Evans RA, Tian YC, Steadman R, Phillips AO. TGF-beta1-mediated fibroblast-myofibroblast terminal differentiation-the role of Smad proteins. Exp Cell Res. 2003; 282: 90-100.

39. Leask A. Transcriptional profiling of the scleroderma fibroblast reveals a potential role for connective tissue growth factor (CTGF) in pathological fibrosis. Keio J Med. 2004; 53: 74-7.

40. Zhang JY, Gao P, Ye W, Xiao YQ. Functional characteristics of connective tissue growth factor on human tenon's capsule fibroblast. Curr Eye Res. 2014; 39: 53-61.

41. Wang S, Li B, Li C, Cui W, Miao L. Potential Renoprotective Agents through Inhibiting CTGF/CCN2 in Diabetic Nephropathy. J Diabetes Res. 2015; 2015: 962383

42. Klaassen I, van Geest RJ, Kuiper EJ, van Noorden CJ, Schlingemann RO. The role of CTGF in diabetic retinopathy. Exp Eye Res. 2015; 133: 37-48.

43. Huang M, Wang YP, Zhu LQ, Cai Q, Li HH, Yang HF. MAPK pathway mediates epithelial-mesenchymal transition induced by paraquat in alveolar epithelial cells. Environ Toxicol. 2016; 31: 140-7.

44. Yang J, Hou Y, Zhou M, Wen S, Zhou J, Xu L, et al. Twist induces epithelial-mesenchymal transition and cell motility in breast cancer via ITGB1-FAK/ILK signaling axis and its associated downstream network. Int J Biochem Cell B. 2016; 71: 62-71.

45. Zeglinski MR, Roche P, Hnatowich M, Jassal DS, Wigle JT, Czubryt MP, et al. TGF $\beta 1$ regulates scleraxis expression in primary cardiac myofibroblasts by a Smad-independent mechanism. Am J Physiol-Heart C. 2015; 39: ajpheart.00584.2015.

46. Li Z, Liu X, Wang B, Nie Y, Wen J, Wang Q, et al. Pirfenidone suppresses MAPK signalling pathway to reverse epithelial-mesenchymal transition and renal fibrosis. Nephrology. 2017; 22: 589-97.

47. Chen B, Li J, Fellows GF, Sun Z, Wang R. Maintaining human fetal pancreatic stellate cell function and proliferation require $\beta 1$ integrin and collagen I matrix interactions. Oncotarget. 2015; 6: 14045-59.
48. Cortina P, Gómez-Lechón MJ, Navea A, Menezo JL, Terencio MC, Diaz-Llopis M. Diclofenac sodium and cyclosporin A inhibit human lens epithelial cell proliferation in culture. Graefes Arch Clin Exp Ophthalmol. 1997; 235: 180-5.

49. Huang X, Wang Y, Cai JP, Ma XY, Li Y, Cheng JW, et al. Sustained release of 5-fluorouracil from chitosan nanoparticles surface modified intra ocular lens to prevent posterior capsule opacification: an in vitro and in vivo study. J Ocul Pharmacol Ther. 2013; 29: 208-15.

50. Guha R, Chowdhury S, Palui H, Mishra A, Basak S, Mandal TK, et al. Doxorubicin-loaded MePEG-PCL nanoparticles for prevention of posterior capsular opacification. Nanomedicine. 2013; 8: 1415-28

51. Davis JL, Yi NY, Salmon JH, Charlton AN, Colitz $\mathrm{CMH}$, Gilger BC. Sustained-Release Celecoxib from Incubated Acrylic Intraocular Lenses Suppresses Lens Epithelial Cell Growth in an Ex Vivo Model of Posterior Capsule Opacity. J Ocul Pharmacol Ther. 2012; 28: 35-9.

52. Wei S, Xu C, Rychak JJ, Luong A, Sun Y, Yang Z, et al. Short Hairpin RNA Knockdown of Connective Tissue Growth Factor by Ultrasound-Targeted Microbubble Destruction Improves Renal Fibrosis. Ultrasound Med Biol. 2016; 42: 2926-37.

53. Shuang L, Lv YF, Su HQ, Zhang QN, Wang LR, Hao ZM. A virus-like particle-based connective tissue growth factor vaccine suppresses carbon tetrachloride-induced hepatic fibrosis in mice. Scientific Reports. 2016; 6: 321-55. 